\title{
Transverse-mode dynamics in vertical-cavity surface-emitting lasers with optical feedback
}

\author{
M. S. Torre, ${ }^{1}$ C. Masoller, ${ }^{2}$ and Paul Mandel ${ }^{3}$ \\ ${ }^{1}$ Instituto de Física "Arroyo Seco," UNCPBA Pinto 399 (7000) Tandil, Argentina \\ ${ }^{2}$ Instituto de Física, Facultad de Ciencias, Universidad de la República, Igua 4225, Montevideo 11400, Uruguay \\ ${ }^{3}$ Université Libre de Bruxelles, Optique Nonlinéaire Théorique, Campus Plaine Code Postale 231, B-1050 Bruxelles, Belgium
}

(Received 22 March 2002, revised manuscript received 21 June 2002; published 26 November 2002)

\begin{abstract}
We study the transverse-mode dynamics of vertical-cavity surface-emitting lasers with weak optical feedback. We use a model that takes into account the spatial dependence of the transverse modes and of two carrier density profiles, associated with confined carriers in the quantum well region of the laser and unconfined carriers in the barrier region. Optical feedback is included as in the Lang-Kobayashi model. We find that for adequate parameter values antiphase dynamics occurs. As the injection current varies, the antiphase dynamics is destroyed through a sequence of periodic mixed states leading to in-phase dynamics. In these mixed states there are time intervals in which the modes are in phase, followed by time intervals in which they are in antiphase. We study the origin of the antiphase dynamics, assessing the role of the different spatial profiles. We show that the competition between the different profiles leads to the observed antiphase behavior.
\end{abstract}

DOI: 10.1103/PhysRevA.66.053817～PACS number(s): 42.60.Mi, 42.55.Px, 42.65.Sf, 05.45.Pq

\section{INTRODUCTION}

The vertical-cavity surface-emitting laser (VCSEL) is a type of semiconductor laser that is emerging as a key element for high-speed information processing systems and optical communication networks [1]. The advantages of a VCSEL over a conventional, edge-emitting semiconductor laser are single-longitudinal-mode operation, dense packing capability, low threshold current, high modulation bandwidth, narrow circular beam profile, and simple and efficient coupling to an optical fiber. Near threshold, VCSELs typically emit linearly polarized light in the fundamental transverse mode. However, it is often observed that the polarization state selected at threshold becomes unstable as the injection current is increased, and a switch to the orthogonal polarization state occurs (see, e.g., Ref. [2] and references therein). For high-power operation, high-order transverse modes are excited and the VCSEL usually emits multiple transverse modes. The complex polarization and transverse-mode behavior of VCSELs are considered drawbacks from the viewpoint of most applications, and have attracted broad interest, both theoretically and experimentally [3-20].

It is well known that optical feedback from an external reflector has important effects on the dynamics of VCSELs [21-27]. The effect of optical feedback depends on the amount of power fed back into the laser cavity, and on the round trip time of the field in the external cavity, which determines the feedback phase. Controlled optical feedback might stabilize the laser, reducing its linewidth, but uncontrolled feedback (unavoidable in many applications) might destabilize the laser, inducing chaotic intensity fluctuations and a broad linewidth. One particularly complex behavior is known as low-frequency fluctuations (LFFs), and is characterized by abrupt random intensity dropouts followed by gradual, deterministic recoveries.

In addition, in multimode lasers optical feedback might induce a variety of complex regimes. Several authors have studied theoretically the dynamics of multiple-longitudinalmode conventional (edge-emitting) semiconductor lasers with optical feedback. Sukow et al. [28] studied the effect of feedback based on an extension of the single-mode LangKobayashi (LK) model [29], which incorporates additional optical modes that are coupled through the carrier inversion and through self- and cross-saturation coefficients. It was found that the statistics of the intensity fluctuations in the LFF regime on a picosecond time scale is essentially independent of the number of optical modes involved in the laser emission. Using a similar model, but with a parabolic gain profile, Rogister et al. [30] showed that in the presence of noise and in the LFF regime two qualitatively different behaviors on the picosecond time scale are possible: the longitudinal modes can emit pulses in phase or oscillate out of phase, depending on the operating parameters. Viktorov and Mandel [31] studied a multimode extension of the LK model that takes into account the longitudinal carrier grating associated with a Fabry-Perot configuration and predicted the possibility of antiphase dynamics. In that model, the steady state is destabilized either by a simple Hopf bifurcation leading to in-phase dynamics of the longitudinal modes, or by a degenerate Hopf bifurcation leading to antiphase dynamics [32].

Antiphase dynamics is an example of collective behavior in a system of globally coupled oscillators [33]. In lasers it results from the phase coherence of time-dependent modal intensities [34,35]. In the simplest cases, it is characterized by the fact that the total intensity, which is the direct sum of the modal intensities for rate equation models, has many properties of the single-mode intensity, while modal intensities display a more complex behavior.

Several studies of the transverse-mode behavior of VCSELs have been based on a model originally proposed by Valle, Sarma, and Shore $[3,4]$. The model includes spatial profiles for the transverse optical modes and for the carrier density in the quantum well (QW) active region of the VCSEL. It also includes carrier diffusion. The model applies to weakly index-guided VCSELs, where the transverse modal profiles and modal frequencies are determined by the built-in refractive index distribution, thus allowing a descrip- 
tion in terms of modal amplitudes.

For a cylindrical VCSEL, the appropriate transverse modes are the linearly polarized $\mathrm{LP}_{m, n}$ modes [36]. They have the property $\operatorname{LP}_{m, n}(r, \theta)=\phi_{m, n}(r) \cos (m \theta)$ where $(r, \theta)$ are the polar coordinates of the plane transverse to the propagation direction. Several authors $[3,22,26,37,38]$ have simplified the numerical simulations by assuming that the azimuthal dependence of the modes with $m>0$ can be neglected, i.e., $\operatorname{LP}_{m, n}(r, \theta) \simeq \phi_{m, n}(r)$, when these modes are degenerate. However, the resulting approximate modes are not cavity modes, except if $m=0$.

In this paper we use a model for VCSELs that is an extension of the model proposed by Valle et al. [3]. However, in order to simplify the calculations while keeping the model as complete as possible, we assume that only the first three azimuthally symmetric modes $\mathrm{LP}_{0,1}, \mathrm{LP}_{0,2}$, and $\mathrm{LP}_{0,3}$ can be excited. Carrier transport effects are included by considering two carrier densities, one for the carriers in the QW region (where the carriers are in two-dimensional quantum states), and one for the carriers in the barrier region (where the carriers are in three-dimensional quantum states). The exchange of carriers between these two reservoirs (carrier capture into the QWs and escape out of the QWs) is characterized by small but finite capture and escape times. Our approach is the same as in the phenomenological standard rate equations for QW lasers [39-41]. External optical feedback is included as in the LK model, by considering a single reflection in the external cavity.

We show that a weak optical feedback may induce antiphase dynamics of the transverse modes, and we study how the antiphase dynamics is destabilized as the injection current or the diffusion coefficient varies. We find that the antiphase dynamics is destroyed through a sequence of periodic mixed states. In these states, time intervals in which the modes are in phase alternate with time intervals in which they are in antiphase. We study the origin of the antiphase behavior by considering equal and different spatial profiles for the transverse modes. We show that it is the competition between the different profiles that leads to the observed antiphase behavior.

The effects of optical feedback on the dynamics of VCSELs were previously studied by Law and Agrawal $[23,24]$, based on a model similar to ours but that takes into account several reflections in the external cavity and does not consider carrier capture and escape. In-phase and antiphase regimes were found in that model but that aspect of the dynamics was not the topic of these papers. Here we focus on studying in detail the in-phase and antiphase behavior. This paper is organized as follows. The model is described in Sec. II. Analytical results for the steady state are presented in Sec. III. Results of numerical simulations that show distinct dynamical regimes of the transverse modes are presented in Sec. IV. Finally, Sec. V contains a summary and the conclusions.

\section{THE MODEL}

We consider a cylindrically symmetric structure, whose active region (consisting of several quantum wells) is mod- eled as a single effective quantum well of radius $a$ and thickness $d_{\mathrm{QW}}$. Barrier regions of thickness $d_{b}$ limit the QW region. Two highly reflecting mirrors separated by a distance $L$ along the longitudinal $z$ axis define the laser cavity. The injected current is azimuthally uniform over the transverse area and varies stepwise: $j(r)=j_{o}$ for $r<a$ and $j(r)=0$ otherwise. The emission behavior is determined by the built-in index guiding introduced by the transverse refractive index step in the surrounding region. The core (cladding) refractive index is taken to be $n_{\text {core }}\left(n_{\text {clad }}\right)$, i.e., the transverse refractive index profile is $n(r)=n_{\text {core }}$ for $r<a$ and $n(r)=n_{\text {clad }}$ for $r>a$. For this geometry the appropriate transverse modes are the linearly polarized $\mathrm{LP}_{m n}$ modes [36], for which the transverse variation of the field is given by

$$
\begin{gathered}
\psi_{m n}(r, \theta)=\frac{J_{m}\left(u_{m n} r / a\right)}{J_{m}\left(u_{m n}\right)} \cos m \theta \text { for } r<a, \\
\psi_{m n}(r, \theta)=\frac{K_{m}\left(w_{m n} r / a\right)}{K_{m}\left(w_{m n}\right)} \cos m \theta \quad \text { for } r>a,
\end{gathered}
$$

where $J_{m}$ and $K_{m}$ are Bessel functions of the first and second kinds, respectively, $u_{m n}=a\left[\left(n_{\text {core }} k_{m n}\right)^{2}-\beta^{2}\right]^{1 / 2}, \quad w_{m n}$ $=a\left[\beta^{2}-\left(n_{\text {clad }} k_{m n}\right)^{2}\right]^{1 / 2}, \beta L=q \pi, q$ is an integer, and the wave vector $k_{m n}$ is obtained from eigenvalue equations. To simplify the calculations we consider that only three modes, having azimuthal symmetry, are excited in the range of parameters considered in this paper:

$$
\begin{gathered}
\psi_{1}(r) \equiv \psi_{01}(r, \theta)=\mathrm{LP}_{01}, \quad \psi_{2}(r) \equiv \psi_{02}(r, \theta)=\mathrm{LP}_{02} \\
\text { and } \psi_{3}(r, \theta) \equiv \psi_{03}(r)=\mathrm{LP}_{03}
\end{gathered}
$$

The mode profiles are normalized such that $\int_{0}^{\infty}\left|\psi_{i}\right|^{2}(r) r d r$ $=1$. Since the mode profiles are exponentially small outside the active region, this normalization hardly differs from the physical normalization $\int_{0}^{a}\left|\psi_{i}\right|^{2}(r) r d r=1$.

The equations for the slowly varying complex amplitude of the $i$ th mode, $e_{i}(t)$, the density of carriers confined in the QW region, $n_{w}(r, t)$, and the density of (unconfined) carriers in the barrier region, $n_{b}(r, t)$, are $[3,29,38]$

$$
\begin{aligned}
\frac{d e_{i}}{d t}= & \frac{1+j \alpha}{2}\left(g_{i}-\frac{1}{\tau_{p i}}\right) e_{i}(t)+k_{i} e_{i}(t-\tau) \exp \left(-j \omega_{i} \tau\right) \\
\frac{\partial n_{b}}{\partial t}= & \frac{j(r)}{e d_{b}}-\frac{n_{b}}{\tau_{c a p}}+\frac{V_{\mathrm{QW}}}{V_{b}} \frac{n_{w}}{\tau_{e s c}}-\frac{n_{b}}{\tau_{n}}+D_{b} \frac{1}{r} \frac{\partial}{\partial r}\left(r \frac{\partial n_{b}}{\partial r}\right) \\
\frac{\partial n_{w}}{\partial t}= & \frac{V_{b}}{V_{\mathrm{QW}}} \frac{n_{b}}{\tau_{c a p}}-\frac{n_{w}}{\tau_{e s c}}-\frac{n_{w}}{\tau_{n}}-g_{o}\left(n_{w}-n_{t}\right) \sum\left|e_{i}\right|^{2}\left|\psi_{i}\right|^{2} \\
& +D_{w} \frac{1}{r} \frac{\partial}{\partial r}\left(r \frac{\partial n_{w}}{\partial r}\right) .
\end{aligned}
$$

In these equations the modal amplitude $e_{i}$ is normalized such that $\left|e_{i}\right|^{2}\left|\psi_{i}\right|^{2}$ is the photon density in the $i$ th mode. The carrier variables are averaged along the longitudinal axis. 
Therefore, $n_{w}(r, t)\left[n_{b}(r, t)\right]$ represents the average carrier density in the transverse plane in the QW (barrier) region. If the QW region consists of several QWs, interwell carrier transport effects are not considered, and $n_{w}$ represents the average carrier density in the QWs.

The first term in the right-hand side of Eq. (3) accounts for optical gain, losses, and phase-amplitude coupling. Here, $\alpha$ is the linewidth enhancement factor and $g_{i}$ is the modal gain,

$$
g_{i}(t)=\int_{0}^{\infty} g_{o} \Gamma_{i}\left(n_{w}-n_{t}\right)\left|\psi_{i}\right|^{2} r d r
$$

where $g_{o}$ is the gain coefficient, $\Gamma_{i}$ is the confinement factor for the $i$ th mode, and $n_{t}$ is the transparency carrier density. $\tau_{p i}$ is the photon lifetime for the $i$ th mode. The second term in the right-hand side of Eq. (3) takes into account the field reflected from the external cavity. We consider a single reflection, and therefore the model is valid for weak and moderate feedback levels. $k_{i}$ is the feedback coefficient of the $i$ th mode: $k_{i}=\left(1-R_{2}\right) \sqrt{R_{2} R_{\text {ext }} \eta_{c}} /\left(R_{2} \tau_{i n}\right)$ [24], where $R_{2}$ and $R_{\text {ext }}$ are the output and external mirror reflectivities, $\tau_{i n}$ is the solitary laser round-trip time, and $\eta_{c}$ is the coupling efficiency. In general $\eta_{c}$ can be different for different transverse modes, but in this study we take $\eta_{c}$ to be mode independent. $\omega_{i}$ is the optical frequency of the $i$ th mode in the absence of feedback, and $\tau$ is the external-cavity round-trip time.

The terms on the right-hand side of Eq. (4) correspond, from left to right, to (i) the rate at which carriers are injected into the barrier region, (ii) the rate at which carriers are captured into the QWs, (iii) the rate at which carriers escape out of the QWs, (iv) the carrier loss owing to various nonradiative recombination processes, and (v) carrier diffusion across the barrier region. The transport effects are included by a capture time $\tau_{c a p}$, an escape time $\tau_{\text {esc }}$, and a diffusion coefficient $D_{b}$. The carrier loss is included by a carrier lifetime $\tau_{n}$. Since the variables $n_{b}$ and $n_{w}$ refer to carrier densities, the different sizes of the barrier and QW regions must be taken into account. This is done by the ratio $V_{b} / V_{\mathrm{QW}}$, where $V_{b}=d_{b} \pi a^{2}$ is the volume of the barrier region, and $V_{\mathrm{QW}}$ $=d_{\mathrm{QW}} \pi a^{2}$ is the volume of the $\mathrm{QW}$ region.

The terms in the right-hand side of Eq. (5) correspond, from left to right, to (i) the carriers captured into the QWs, (ii) the carriers that escape out of the QWs, (iii) the nonradiative carrier loss, (iv) the carrier loss owing to stimulated recombination, and (v) carrier diffusion across the QWs. For simplicity we consider the same nonradiative recombination time for the carriers in the QW region and for the carriers in the barrier region (the effect of different recombination times was studied in [38]).

\section{STEADY-STATE SOLUTIONS}

The stationary solutions of Eqs. (3)-(5) are

$$
\begin{gathered}
e_{i}(t)=e_{i}^{s} \exp \left[i\left(\omega_{i}^{s}-\omega_{i}\right) t\right], \\
n_{w}(r, t)=n_{w}^{s}(r), \quad n_{b}(r, t)=n_{b}^{s}(r),
\end{gathered}
$$

where $e_{i}^{s}, \omega_{i}^{s}, n_{w}^{s}(r)$, and $n_{b}^{s}(r)$ satisfy

$$
\begin{aligned}
& g_{i}^{s}=\int_{0}^{\infty} g_{o} \Gamma_{i}\left(n_{w}^{s}-n_{t}\right)\left|\psi_{i}\right|^{2} r d r=1 / \tau_{p i}-2 k_{i} \cos \left(\omega_{i}^{s} \tau\right), \\
& \omega_{i}^{s}-\omega_{i}=-\alpha k_{i} \cos \left(\omega_{i}^{s} \tau\right)-k_{i} \sin \left(\omega_{i}^{s} \tau\right), \\
& n_{b}^{s}\left(\frac{1}{\tau_{c a p}}+\frac{1}{\tau_{n}}\right)=\frac{j(r)}{e d_{b}}+\frac{V_{\mathrm{QW}}}{V_{b}} \frac{n_{w}^{s}}{\tau_{e s c}}+D_{b} \frac{1}{r} \frac{\partial}{\partial r}\left(r \frac{\partial n_{b}^{s}}{\partial r}\right), \\
& n_{w}^{s}\left(\frac{1}{\tau_{e s c}}+\frac{1}{\tau_{n}}\right)=\frac{V_{b}}{V_{\mathrm{QW}}} \frac{n_{b}^{s}}{\tau_{c a p}}-g_{o}\left(n_{w}^{s}-n_{t}\right) \sum\left|e_{i}^{s}\right|^{2}\left|\psi_{i}\right|^{2} \\
& +D_{w} \frac{1}{r} \frac{\partial}{\partial r}\left(r \frac{\partial n_{w}^{s}}{\partial r}\right) \text {. }
\end{aligned}
$$

Equation (7) shows that the stationary values of the modal gains depend on the feedback level but not on the carrier capture and escape times. Equation (8) determines the optical frequencies of the transverse modes in the presence of feedback, which are also independent of $\tau_{c a p}$ and $\tau_{e s c}$. Integrating Eqs. (9) and (10) between $r=0$ and $r=\infty$ gives

$$
\begin{aligned}
\left(\gamma_{c a p}+\gamma_{n}\right) \int_{0}^{\infty} n_{b}^{s} r d r= & \frac{1}{e d_{b}} \int_{0}^{\infty} j(r) r d r+\frac{V_{\mathrm{QW}}}{V_{b}} \gamma_{e s c} \int_{0}^{\infty} n_{w}^{s} r d r \\
& +D_{b} \int_{0}^{\infty} \frac{1}{r} \frac{\partial}{\partial r}\left(r \frac{\partial n_{b}^{s}}{\partial r}\right) r d r \\
\left(\gamma_{e s c}+\gamma_{n}\right) \int_{0}^{\infty} n_{w}^{s} r d r= & \frac{V_{b}}{V_{\mathrm{QW}}} \gamma_{c a p} \int_{0}^{\infty} n_{b}^{s} r d r \\
& -\sum\left|e_{i}^{s}\right|^{2} \int_{0}^{\infty} g_{o}\left(n_{w}^{s}-n_{t}\right)\left|\psi_{i}\right|^{2} r d r \\
& +D_{w} \int_{0}^{\infty} \frac{1}{r} \frac{\partial}{\partial r}\left(r \frac{\partial n_{w}^{s}}{\partial r}\right) r d r
\end{aligned}
$$

where $\gamma_{e s c}=1 / \tau_{e s c}, \gamma_{c a p}=1 / \tau_{c a p}$, and $\gamma_{n}=1 / \tau_{n}$. The numbers of carriers in the barrier and QW regions are

$$
\begin{gathered}
N_{b}(t)=2 \pi d_{b} \int_{0}^{\infty} n_{b}(r, t) r d r, \\
N_{w}(t)=2 \pi d_{\mathrm{QW}} \int_{0}^{\infty} n_{\mathrm{QW}}(r, t) r d r,
\end{gathered}
$$

and Eqs. (11) and (12) can be rewritten as

$$
\begin{aligned}
\left(\gamma_{c a p}+\gamma_{n}\right) N_{b}^{s}= & \frac{2 \pi}{e} \int_{0}^{\infty} j(r) r d r+\gamma_{e s c} N_{w}^{s} \\
& +\left.D_{b} 2 \pi d_{b} r \frac{\partial n_{b}^{s}}{\partial r}\right|_{r=0} ^{r=\infty},
\end{aligned}
$$




$$
\begin{aligned}
\left(\gamma_{e s c}+\gamma_{n}\right) N_{w}^{s}= & \gamma_{c a p} N_{b}^{s}-2 \pi d_{\mathrm{QW}} \sum\left|e_{i}^{s}\right|^{2} g_{i}^{s} / \Gamma_{i} \\
& +\left.D_{w} 2 \pi d_{\mathrm{QW}} r \frac{\partial n_{w}^{s}}{\partial r}\right|_{r=0} ^{r=\infty},
\end{aligned}
$$

where $\quad N_{b}^{s}=2 \pi d_{b} \int_{0}^{\infty} n_{b}^{s}(r) r d r$ and $N_{w}^{s}$ $=2 \pi d_{\mathrm{QW}} \int_{0}^{\infty} n_{\mathrm{QW}}^{s}(r) r d r$. Now it is clear why it is convenient to define the normalization condition and all integrals between $r=0$ and $r=\infty . n_{w}(r=\infty)=n_{b}(r=\infty)=0$ and the diffusion terms vanish. Equations (13) and (14) can be simplified to

$$
\begin{gathered}
\left(\gamma_{c a p}+\gamma_{n}\right) N_{b}^{s}=J+\gamma_{e s c} N_{w}^{s}, \\
\left(\gamma_{e s c}+\gamma_{n}\right) N_{w}^{s}=\gamma_{c a p} N_{b}^{s}-\gamma_{p} I_{T},
\end{gathered}
$$

where $J=2 \pi \int_{0}^{\infty} j(r) r d r / e$ is the number of injected carriers per unit time, and $I_{T}=2 \pi d_{\mathrm{QW}} \Sigma\left|e_{i}^{s}\right|^{2}$ is the total number of photons in the QW region. In Eq. (16) we have assumed that $g_{i}^{s} \sim 1 /\left(\Gamma_{i} \tau_{p i}\right)=\gamma_{p}$. This approximation is valid for low feedback levels such that $1 / \tau_{p i} \gg k_{i}$.

From Eqs. (15) and (16) we can eliminate $N_{b}^{s}$ and obtain

$$
\gamma_{p} I_{T}=\frac{J}{1+\gamma_{n} / \gamma_{c a p}}-\frac{\gamma_{n} N_{w}^{s}}{1+\gamma_{n} / \gamma_{c a p}}\left(1+\frac{\gamma_{n}}{\gamma_{c a p}}+\frac{\gamma_{e s c}}{\gamma_{c a p}}\right) \text {. }
$$

Two limits are interesting to analyze. When the carriers do not escape out of the QW region $\left(\gamma_{e s c} \sim 0\right)$, we recover the simple connection between the photon number, the injected current, and the carrier number in the QW region, $\gamma_{p} I_{T}=J_{e f f}-\gamma_{n} N_{w}^{s}$, with a modified, "effective" injected current $J_{\text {eff }}=J\left(1+\gamma_{n} / \gamma_{\text {cap }}\right)^{-1}$, which is slightly lower than the actual injected current (typically, $\tau_{n}$ is of the order of nanoseconds, and $\tau_{\text {cap }}$ is of the order of picoseconds; thus $\left.\gamma_{n} / \gamma_{\text {cap }} \ll 1\right)$. The factor $\left(1+\gamma_{n} / \gamma_{\text {cap }}\right)^{-1}$ represents the loss of carriers (due to nonradiative processes) during the capture time. In other words, a finite capture time slightly diminishes the current density effectively injected into the QW region. The other limit corresponds to a large ratio between the capture and the escape times, $R=\tau_{c a p} / \tau_{e s c}=\gamma_{e s c} / \gamma_{c a p}$. This leads to a larger, negative contribution of the last term in Eq. (17), and, therefore, to a significant reduction of the total number of photons in the QW region. We will show in the next section that this favors emission in the fundamental transverse mode.

A complementary way to understand the effect of carrier capture and escape is by considering the dependence of the threshold current of the fundamental transverse mode on the capture and escape times. The threshold current can be estimated from Eq. (17) as

$$
J_{t h}=\gamma_{n} N_{w}^{s}\left(1+\frac{\gamma_{n}}{\gamma_{c a p}}+\frac{\gamma_{e s c}}{\gamma_{c a p}}\right) .
$$

Clearly, an increase of $\gamma_{e s c}$ increases the threshold current, and, therefore, for a fixed injection current the laser operates closer to threshold.

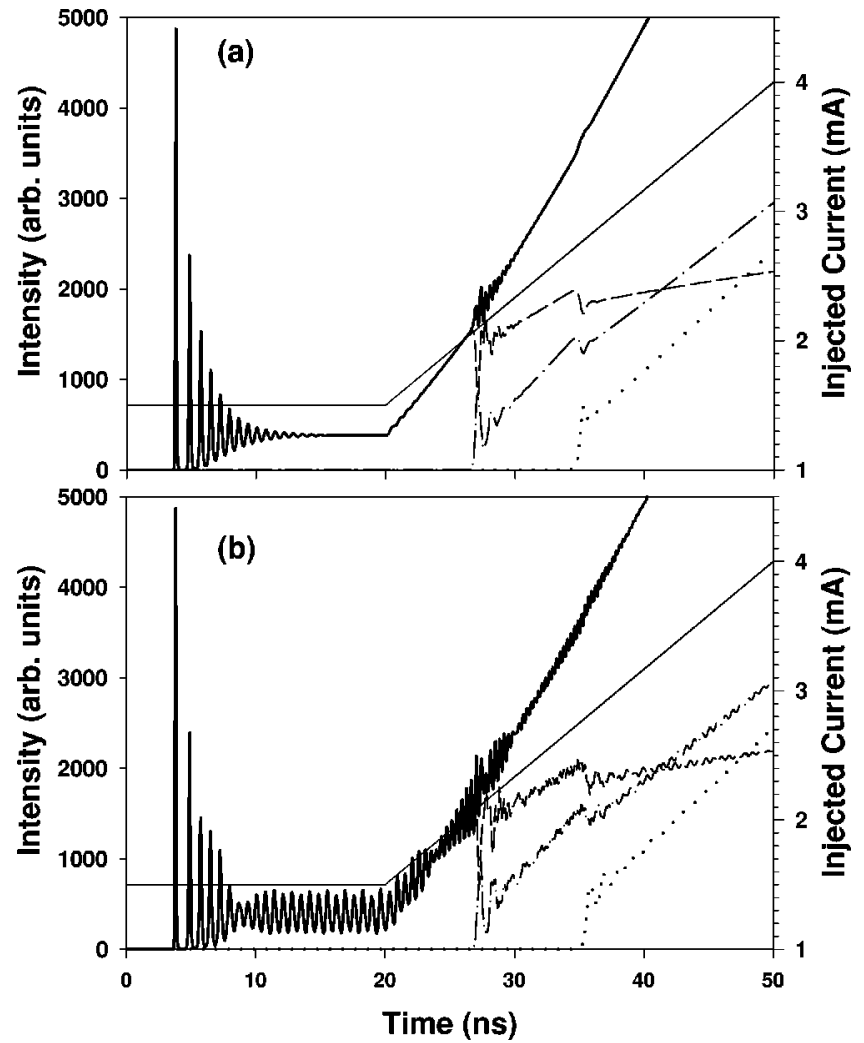

FIG. 1. Total and modal intensities as the injection current increases. The diffusion coefficient is $D_{w}=0.5 \mu \mathrm{m}^{2} / \mathrm{ns}$. (a) Without feedback. (b) The feedback level is $k=1 \mathrm{~ns}^{-1}$. The thick (thin) line shows the value of the total intensity (injection current). The modes are represented as $\mathrm{LP}_{01}$, dashed line; $\mathrm{LP}_{02}$, dot-dashed line; $\mathrm{LP}_{03}$, dotted line.

\section{DYNAMICAL REGIMES}

We integrated the model equations with the parameters $a=6 \mu \mathrm{m}, d_{\mathrm{QW}}=0.024 \mu \mathrm{m}$ (three QWs each of thickness $0.08 \mu \mathrm{m}), \quad d_{b}=1.2 \mu \mathrm{m}, \quad$ index $\mathrm{step}=0.1, \quad \alpha=3, \quad g_{o}$ $=v_{g} \partial g / \partial n$ with $v_{g}=0.0715 \mu \mathrm{m} / \mathrm{ns}$ and $\partial g / \partial n=5.95$ $\times 10^{-8} \mu \mathrm{m}^{2}, \quad n_{t}=1.33 \times 10^{6} \mu \mathrm{m}^{-3}, \quad \tau_{\text {cap }}=5 \mathrm{ps}, \quad \tau_{\text {esc }}$ $=25.5 \mathrm{ps}, \tau_{n}=1.52 \mathrm{~ns}, \tau=1 \mathrm{~ns}$, and $D_{b}=0.5 \mu \mathrm{m}^{2} / \mathrm{ns}$. The time integration step is $\Delta t=10^{-4}$ ps and the space integration step is $\Delta r=0.02 \mu \mathrm{m}$. First, we consider a degenerate situation, in which all modes have the same confinement factor $\Gamma_{i}=0.038$, frequency $\left(\omega_{i} \tau=0 \mathrm{rad}\right)$, losses $\left(\tau_{p i}\right.$ $=2.2 \mathrm{ps})$, and feedback level $\left(k_{i}=k\right)$. The feedback level, the diffusion coefficient $D_{w}$, and the injection current $I$ $=j_{o} \pi a^{2}$ are the free parameters of our study. We show the existence of an antiphase dynamic regime for weak feedback and adequate parameter values. Next, we study the effect of carrier diffusion and modal profiles on the antiphase regime. Finally, we show that the antiphase regime is also observed in a more realistic situation, in which the modes have different optical frequencies.

Without feedback and close to threshold, the single-mode steady state is stable, while for larger injection the transverse multimode steady state is stable. The fundamental $\mathrm{LP}_{01}$ transverse mode has the lowest threshold and it is stable for low current. As the current increases the $\mathrm{LP}_{02}$ mode turns on. 


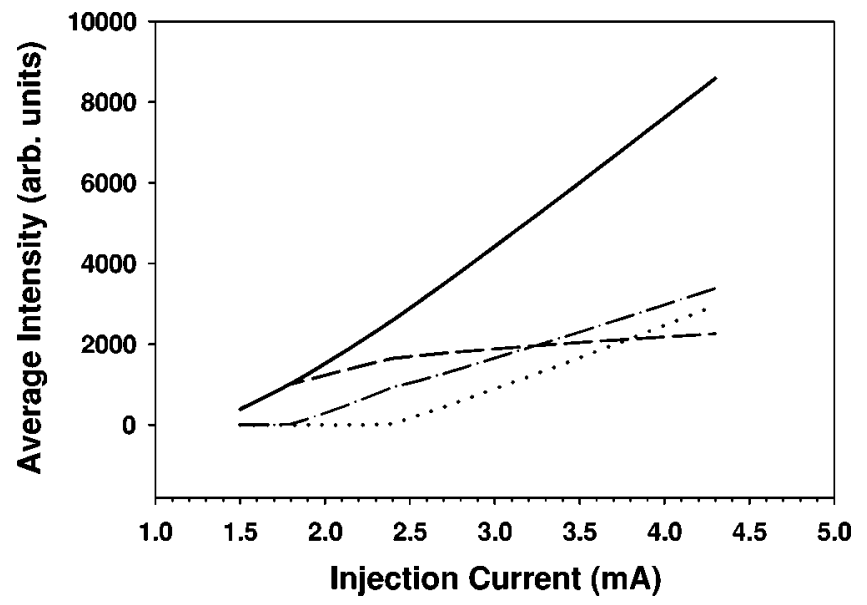

FIG. 2. Total and modal averaged intensities as a function of the injection current. All parameters are as in Fig. 1(b). The thick line shows the value of the total intensity. The modal intensities are represented as $\mathrm{LP}_{01}$, dashed line; $\mathrm{LP}_{02}$, dot-dashed line; $\mathrm{LP}_{03}$, dotted line.

For even larger injection, the $\mathrm{LP}_{03}$ mode turns on and the three modes coexist. Figure 1(a) displays the total and modal intensities in the absence of feedback, as the injection current (thin solid line) gradually increases. The thick line corresponds to the total power, while the other lines correspond to the modal powers $\left(\mathrm{LP}_{01}\right.$ dashed line; $\mathrm{LP}_{02}$ dot-dashed line; $\mathrm{LP}_{03}$ dotted line).

Weak feedback levels modify this picture quantitatively but not qualitatively. Figure 1(b) corresponds to $k=1 \mathrm{~ns}^{-1}$. Considering the internal round trip $\tau_{i n}=0.045 \mathrm{ps}$, the coupling efficiency $\eta_{c}=1$, and the output-mirror reflectivity $R_{2}=0.995$, this feedback level corresponds to an externalmirror reflectivity of $R_{\text {ext }}=8.06 \times 10^{-5}$, i.e., we work in the very weak feedback regime. Figure 1(b) shows that for this feedback the modal intensities exhibit oscillations, and we find distinct dynamical regimes with increasing current. To investigate in more detail what happens for different injection currents, we plot in Fig. 2 the time-averaged value of the total and modal intensities, as a function of the injection current. In this figure the injection current $I$ was kept constant until the stable regime was reached, and then the average value of the total and modal intensities was calculated. Clearly, with weak feedback the transverse-mode dynamics is such that the total intensity looks single mode, i.e., it increases linearly with the injection current except for the weak nonlinear response close to threshold. To display another facet of this intriguing coherence, we show in Fig. 3 the maximum and minimum values of the total [Fig. 3(a)] and the modal [Fig. 3(b)] intensities whose time average is shown in Fig. 2. Figure 3 reveals a complex underlying transverse-mode behavior, which is typical of antiphase dynamics in globally coupled nonlinear oscillators.

\section{A. Antiphase dynamics}

For $I \leqslant 1.7 \mathrm{~mA}$ the VCSEL is single mode. The intensity of the $\mathrm{LP}_{01}$ mode exhibits undamped relaxation oscillations, whose amplitude decreases for increasing I. Figure 3 indi-
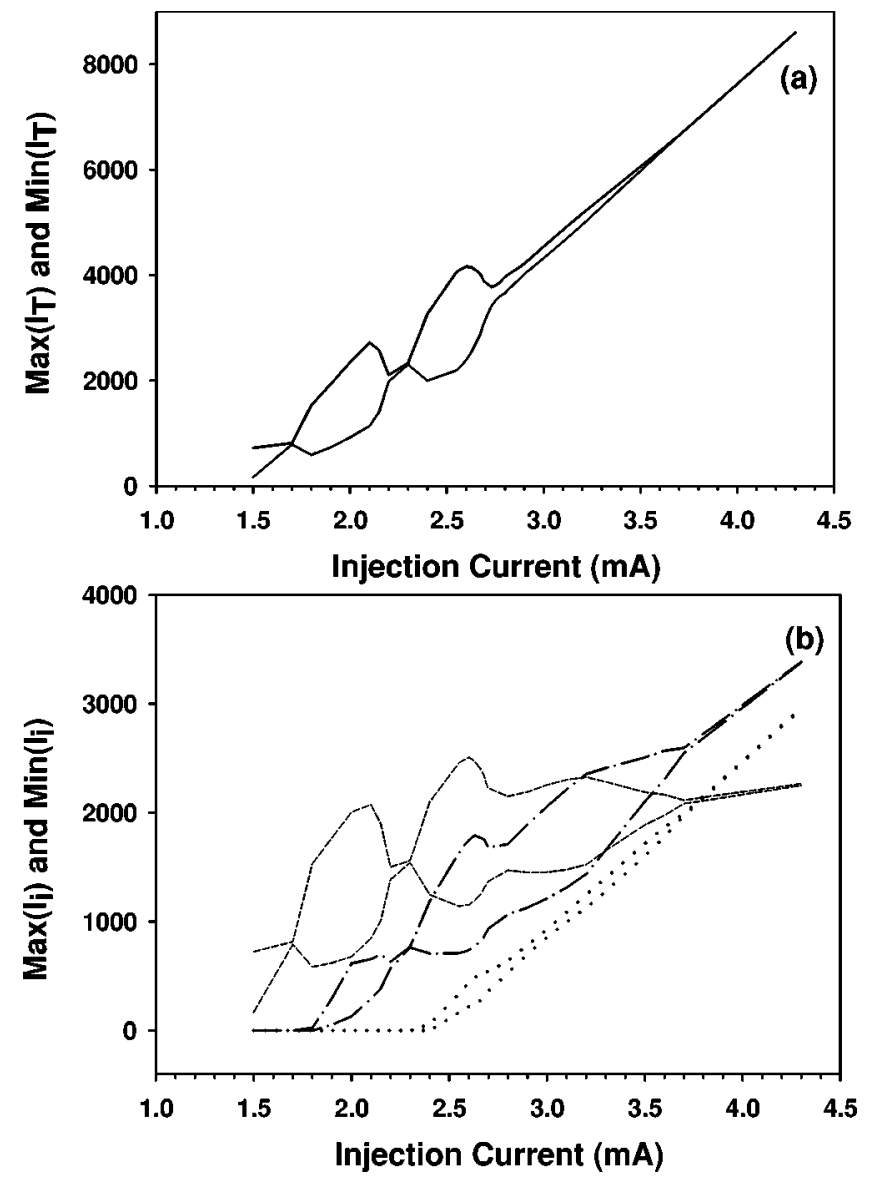

FIG. 3. Maximum and minimum values of the (a) total and (b) modal intensities as a function of the injection current. All parameters are as in Fig. 1(b). The modal intensities are represented as $\mathrm{LP}_{01}$, dashed line; $\mathrm{LP}_{02}$, dot-dashed line; $\mathrm{LP}_{03}$, dotted line.

cates that for $I=1.7 \mathrm{~mA}$ there is single-mode steady-state operation involving only the $\mathrm{LP}_{01}$ mode. For $I$ slightly larger, the $\mathrm{LP}_{02}$ mode emerges, destabilizing the steady-state $\mathrm{LP}_{01}$ mode. In the interval $1.7<I<2.2 \mathrm{~mA}$, the two modes oscillate in phase. As the current is increased, a two-mode steady state is reached for $I=2.3 \mathrm{~mA}$. As for the case $I=1.7 \mathrm{~mA}$, increasing $I$ destabilizes the steady-state operation via the emergence of a new mode. For $I=2.4 \mathrm{~mA}$ the $\mathrm{LP}_{03}$ mode emerges and for $I>2.4 \mathrm{~mA}$ we observe different regimes of three-mode operation. First, for $2.4<I<2.7 \mathrm{~mA}$ there are oscillations of the total intensity, since the maximum and minimum values differ [Fig. 3(a)]. For $I>2.7 \mathrm{~mA}$ the maximum and minimum values of the total intensity are nearly equal, and Fig. 3(b) shows that two distinct dynamical regimes actually occur. For $2.7<I<3.7 \mathrm{~mA}$ there are oscillations of the modal intensities which nearly compensate in the total intensity, while for $I \geqslant 3.7 \mathrm{~mA}$ each transverse mode is in steady state.

Figures 3(a) and 3(b) suggest that in the interval $2.7<I$ $<3.7 \mathrm{~mA}$ antiphase dynamics occurs, since the modal intensities oscillate while the total intensity remains nearly constant. In order to study the phase relations among the modes in the different dynamical regimes, Fig. 4 shows for increasing values of the injection current the total and modal inten- 


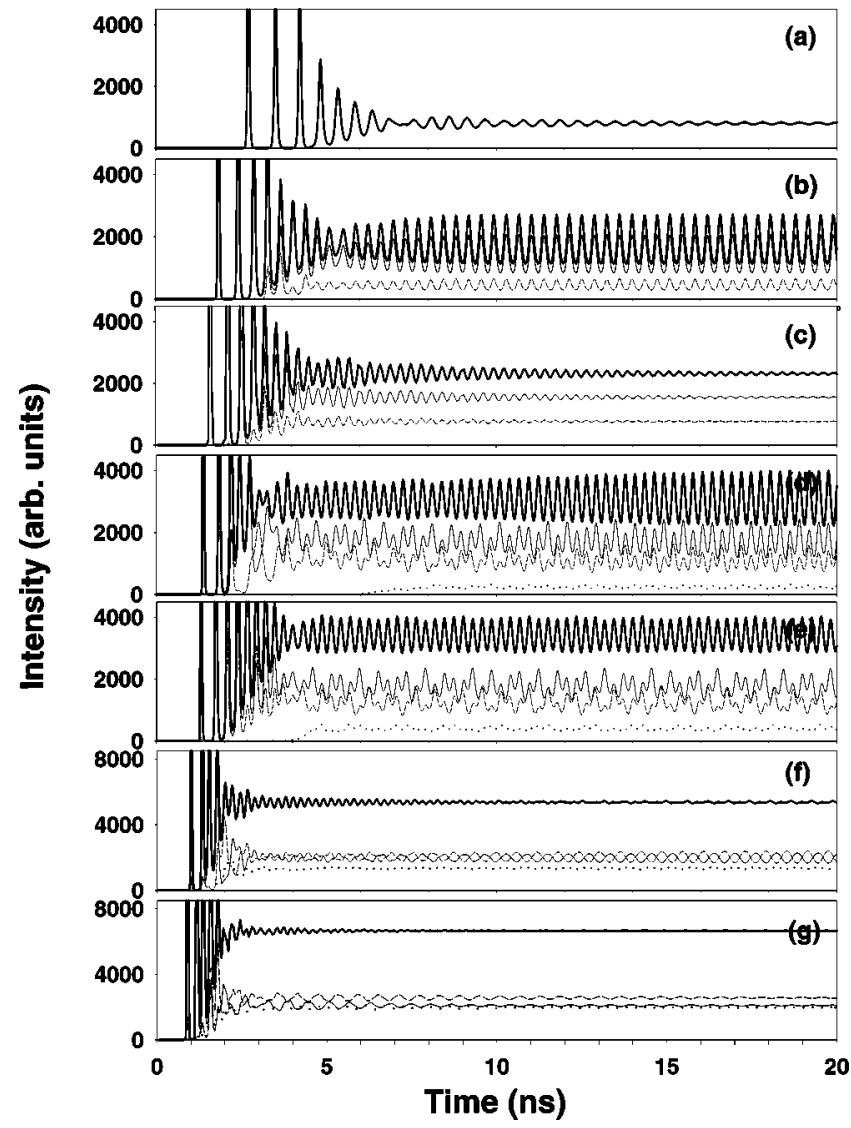

FIG. 4. Dynamic regimes for increasing injection current. (a) $I$ $=1.7 \mathrm{~mA}$; (b) $I=2.1 \mathrm{~mA}$; (c) $I=2.3 \mathrm{~mA}$; (d) $I=2.55 \mathrm{~mA}$; (e) $I$ $=2.67 \mathrm{~mA}$; (f) $I=3.3 \mathrm{~mA}$; (g) $I=3.7 \mathrm{~mA}$. All other parameters are as in Fig. 1(b).

sities. As before, the thick line shows the total power, while the thin lines show the modal powers $\left(\mathrm{LP}_{01}\right.$, dashed line; $\mathrm{LP}_{02}$, dot-dashed line; $\mathrm{LP}_{03}$, dotted line). For low current there is the single-mode periodic regime shown in Fig. 4(a). Figure 4(b) displays a two-mode in-phase regime with very different oscillation amplitudes. Figure 4(c) corresponds to a two-mode steady-state regime. The emergence of the third mode leads to in-phase oscillations for the other two modes, Fig. 4(d). The transition to antiphase oscillations, Fig. 4(f), is through a mixed state displayed in Fig. 4(e). Note that in Fig. 4(e) there are time intervals in which the $\mathrm{LP}_{01}$ and $\mathrm{LP}_{02}$ mode pulses are in phase followed by time intervals in which they are in antiphase. As a whole, this regime is periodic. For even larger injections a three-mode steady state is reached [Fig. 4(g)].

Antiphase behavior was also found by Valle [42], in the competition of the $\mathrm{LP}_{11}^{c}$ [with a $\cos ^{2}(\theta)$ dependence intensity profile], and the $\operatorname{LP}_{11}^{s}$ [with a $\sin ^{2}(\theta)$ dependence intensity profile], when the VCSEL is subjected to injection current modulation. Moreover, previous studies by Law and Agrawal $[23,24]$ of the dynamics of VCSELs with feedback (based on a model similar to ours, but that includes several reflections in the external cavity and does not take into account carrier capture and escape) revealed the existence of in-phase and antiphase behavior. In $[23,24]$ the authors considered high

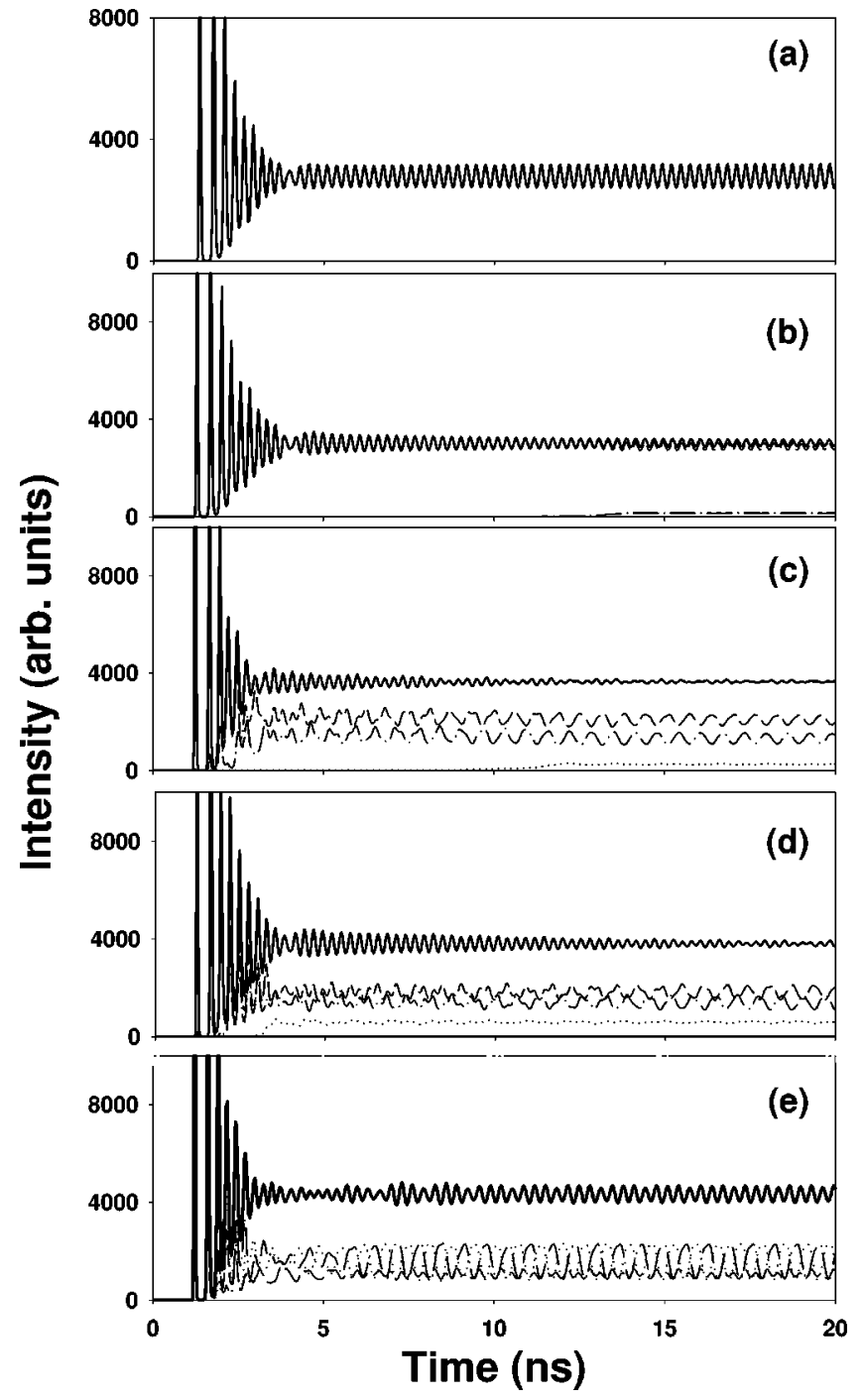

FIG. 5. Dynamic regimes for decreasing carrier diffusion. $k$ $=1 \mathrm{~ns}^{-1}, \quad I=2.8 \mathrm{~mA}$. (a) $D_{w}=3.0 \mu \mathrm{m}^{2} / \mathrm{ns} ; \quad$ (b) $D_{w}$ $=2.0 \mu \mathrm{m}^{2} / \mathrm{ns}$; (c) $D_{w}=1.5 \mu \mathrm{m}^{2} / \mathrm{ns}$; (d) $D_{w}=0.5 \mu \mathrm{m}^{2} / \mathrm{ns}$; (e) $D_{w}=0.01 \mu \mathrm{m}^{2} / \mathrm{ns}$.

reflectivity, the competition of only two transverse modes, and different contact geometries for the injection current. For instance, with a disk-contact geometry, periodic in-phase and antiphase behaviors of the $\mathrm{LP}_{01}$ and $\mathrm{LP}_{11}$ modes were found (see Figs. 5b and 5c of [23]) for different values of the feedback parameter. Thus, antiphase dynamics seems to be a robust general feature independent of the details of the model.

As discussed in Sec. III, the effect of carrier capture and escape is to modify the current effectively injected into the laser cavity. Our numerical simulations verify that increasing $\tau_{e s c}$ is indeed equivalent to increasing the injection current, and a transition from in-phase to antiphase behavior can be observed. In the following sections we study the influence of carrier diffusion, modal profiles, and different optical frequencies on the antiphase behavior.

\section{B. Influence of the carrier diffusion}

Varying the diffusion coefficient also changes the threshold current, and therefore decreasing $D_{w}$ has an effect similar 


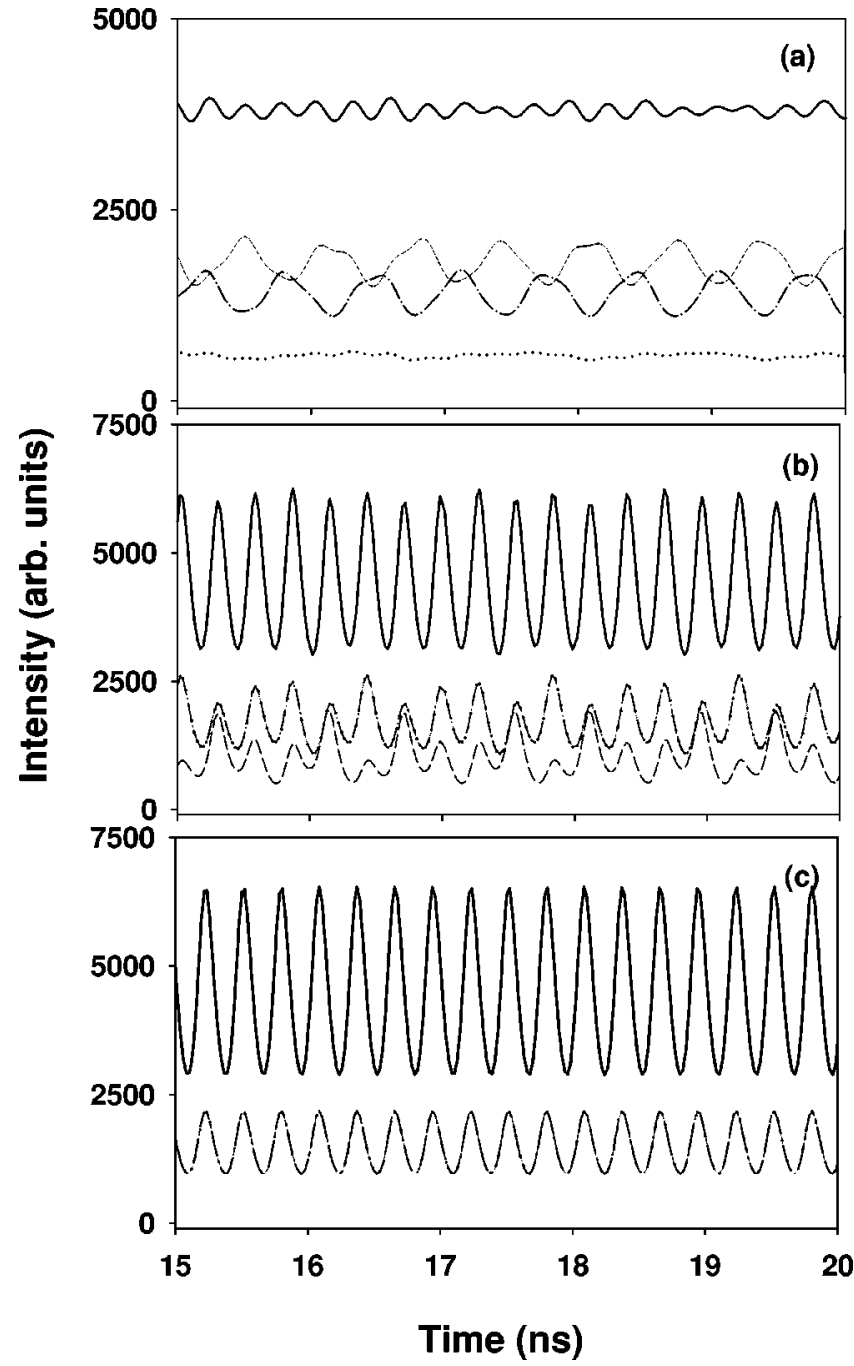

FIG. 6. Effect of different transverse mode profiles. $k$ $=1 \mathrm{~ns}^{-1}, I=2.8 \mathrm{~mA}, D_{w}=0.5 \mu \mathrm{m}^{2} / \mathrm{ns}$. (a) The transverse modes are $\mathrm{LP}_{01}, \mathrm{LP}_{02}$, and $\mathrm{LP}_{03}$. (b) Two transverse modes have the same uniform profiles, and the third mode is the $\mathrm{LP}_{01}$ mode. (c) The three transverse modes have the same uniform profiles.

to increasing the injection. Figure 5 shows the transversemode dynamics for five decreasing values of the diffusion coefficient. For large diffusion [Fig. 5(a)] the laser operates on the fundamental transverse mode in a periodic regime. As the diffusion coefficient decreases, we observe a transition to antiphase oscillations involving the $\mathrm{LP}_{01}$ and $\mathrm{LP}_{02}$ modes [Figs. 5(c) and 5(d)]. As the diffusion coefficient is further decreased, we find a more complex type of antiphase regime: antiphase oscillations involving the $\mathrm{LP}_{02}$ and $\mathrm{LP}_{03}$ modes, while the $\mathrm{LP}_{01}$ mode exhibits small oscillations that are in phase, alternately with the $\mathrm{LP}_{02}$ and the $\mathrm{LP}_{03}$ modes [Fig. $5(\mathrm{e})]$. The results obtained for different values of the diffusion coefficient clearly show that in this model it is the population grating due to the Fabry-Perot configuration that induces the multimode behavior, as occurs in other types of homogeneously broadened lasers.

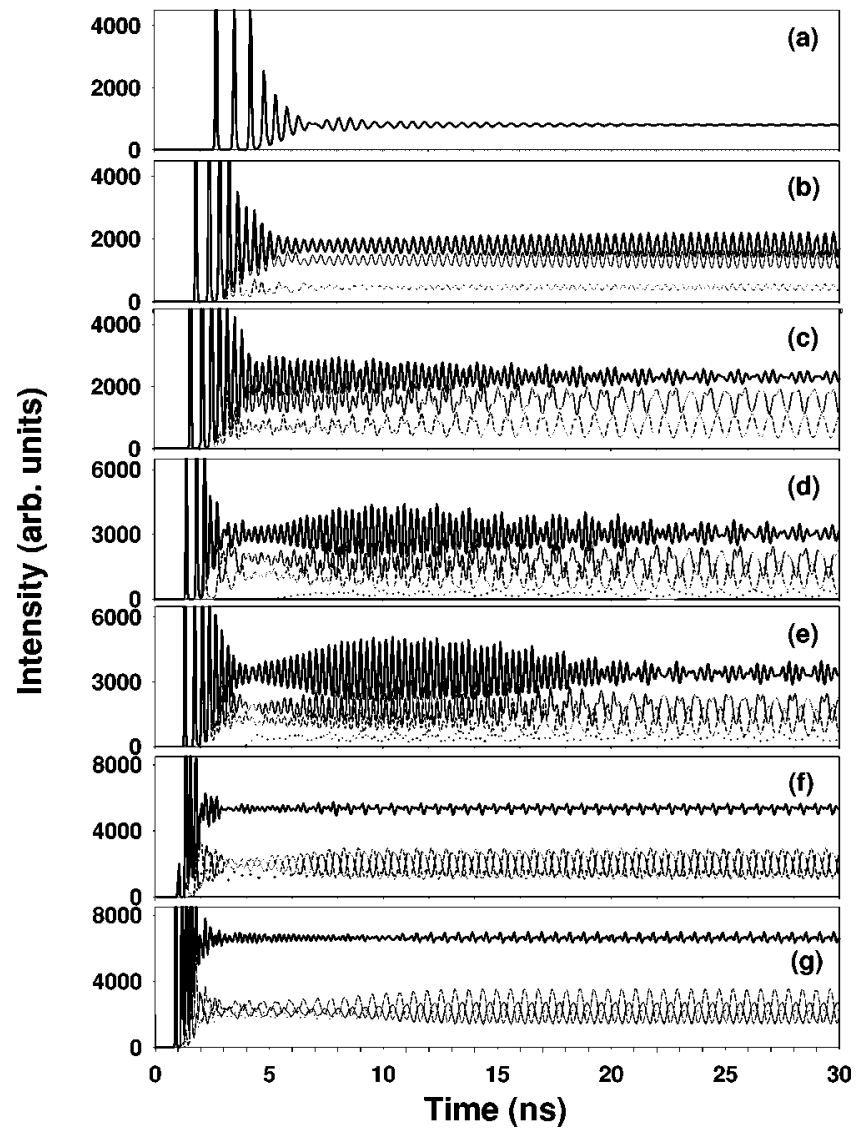

FIG. 7. Dynamic regimes for increasing injection current, when the transverse modes have different optical frequencies. (a) $I$ $=1.7 \mathrm{~mA}$; (b) $I=2.1 \mathrm{~mA}$; (c) $I=2.3 \mathrm{~mA}$; (d) $I=2.55 \mathrm{~mA}$; (e) $I$ $=2.67 \mathrm{~mA}$; (f) $I=3.3 \mathrm{~mA}$; (g) $I=3.7 \mathrm{~mA} . \lambda_{1}=851.8 \mathrm{~nm}, \lambda_{2}$ $=852.0 \mathrm{~nm}$, and $\lambda_{3}=852.2 \mathrm{~nm}$. All other parameters are as in Fig. 4.

\section{Transverse profile competition}

In order to understand the origin of antiphase dynamics in this model, we analyze the competition among the three transverse modes. For that purpose, we shall replace the LP profiles used up to here [Eq. (1)] by other profiles.

Figure 6 shows the effect of different profiles. Figure 6(a) is used as a reference: the transverse profiles are the $\mathrm{LP}_{01}$, $\mathrm{LP}_{02}$, and $\mathrm{LP}_{03}$ modes, and an antiphase oscillation is observed between the $\mathrm{LP}_{01}$ and $\mathrm{LP}_{02}$ modes. Next, we assume that modes 2 and 3 have uniform profiles within the active region $\left[\left|\psi_{i}(r)\right|^{2}=c_{i}\right.$ for $r<a$ and $\left|\psi_{i}(r)\right|^{2}=0$ otherwise] while the third mode is the fundamental transverse mode $\mathrm{LP}_{01}$. This case is shown in Fig. 6(b), where we observe that the two modes with equal profiles are identical (dot-dashed line), while the third mode (dashed line) oscillates such that large (small) peaks in the identical modes correspond to small (large) peaks in the third mode. Last, we consider the case in which all modes have uniform profiles. In this case, all three modes are identical and oscillate in phase, and the total intensity is exactly three times the intensity of any mode [Fig. 6(c)].

The initial conditions are the same in Figs. 6(a), 6(b), and 6(c). The initial conditions are taken all through the paper 
with the laser off, i.e., the modal amplitudes are at the noise level and the carrier densities are at the transparency value. The different dynamic regimes shown in Figs. 6(a), 6(b), and 6(c) are thus a consequence of the transverse-mode profiles considered.

From these results it is clear that in this model the antiphase dynamics has its origin in the mode coupling and competition (fourth term) in Eq. (5). The competition among the transverse modes with different spatial profiles to "burn holes" in the same reservoir of carriers (i.e., the carriers in the QWs) leads to the observed antiphase dynamics. Equivalently, one can interpret this mechanism as a transverse carrier grating induced by the different weights $\left|\psi_{i}\right|^{2}$ of the lasing modes. This behavior has also been found recently in the dynamics of the longitudinal modes of an edge-emitting laser with optical feedback $[31,32]$ and it is the same mechanism that leads to antiphase dynamics.

\section{Influence of different optical frequencies and different feedback levels}

In the preceding subsections, the three transverse modes have the same optical frequencies, and one might question if the antiphase regime found is not a singular property of the degenerate equations. Figure 7 shows results in which the modes have different optical frequencies: $\lambda_{1}=851.8 \mathrm{~nm}$, $\lambda_{2}=852.0 \mathrm{~nm}$, and $\lambda_{3}=852.2 \mathrm{~nm}$, all other parameters being as in Fig. 4. The antiphase regime persists, and this confirms that, given the smallness of the intermode frequency separation with respect to the optical frequency, intermode frequency differences may be treated by a perturbation theory of which Eqs. (3)-(5) are the zero order approximation.

The antiphase regime is also robust with respect to slightly different feedback levels. Figure 8 shows results for $k_{1}=k_{2}=1 \mathrm{~ns}^{-1}, k_{3}=2 \mathrm{~ns}^{-1}$, all other parameters being as in Fig. 4(f). Clearly the antiphase regime survives.

\section{SUMMARY AND CONCLUSIONS}

The transverse-mode dynamics of an index-guided vertical-cavity surface-emitting laser with weak optical feedback was analyzed using a model that takes into account three transverse modes and two carrier density profiles, associated with confined carriers in the quantum well region of the laser. We found antiphase dynamics and studied the destabilization of this regime when the injection current or the carrier diffusion varies. We found that the antiphase dynamics is destabilized though a sequence of mixed states, in

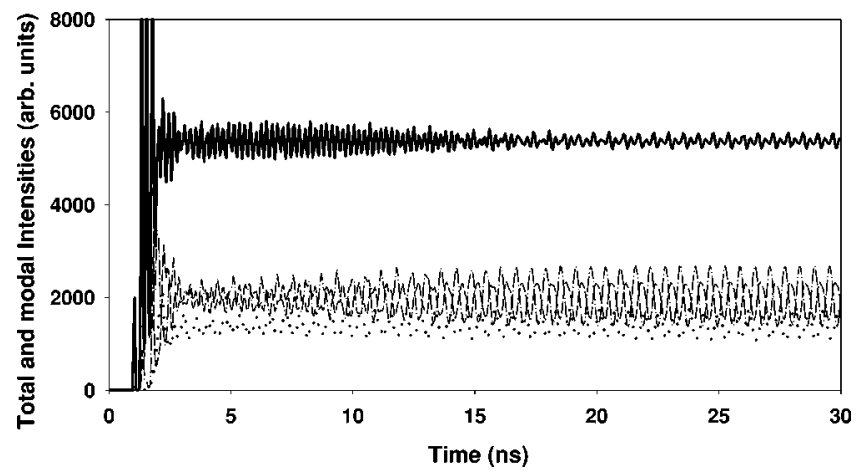

FIG. 8. Antiphase regime when the modes have different feedback levels. $k_{1}=1 \mathrm{~ns}^{-1}, k_{2}=1 \mathrm{~ns}^{-1}$, and $k_{3}=2 \mathrm{~ns}^{-1}$. All other parameters are as in Fig. 4(b).

which time intervals in which the mode pulses are in phase alternate with time intervals in which they are in antiphase. We have also shown that in this model the antiphase dynamics is due to the transverse profiles of the optical modes, which compete for the same reservoir of carriers.

Antiphase dynamics is usually studied in the frame of modal rate equations [30-32], where the carrier density is expanded either in Fourier series or in modal series. These series have to be truncated and the approximation induced by this truncation is difficult to assess [43]. The power of the model studied in this paper is that no such expansion has been introduced and the carrier density dynamical equation is complete, including diffusion. As a result, there is no ambiguity as to the origin of the antiphase dynamics. This is especially clear from the analysis of Sec. IV C, where antiphase dynamics was clearly attributed to the transverse grating of the carriers. The negative aspect of this model is that little can be concluded analytically about the timedependent regimes of the model equations (3)-(5). Numerical simulations are essential to understand the dynamical regimes.

\section{ACKNOWLEDGMENTS}

M.S.T. was supported in part by a grant from Secretaría de Ciencia y Técnica (UNCPBA-Argentina), C.M. was supported in part by Proyecto de Desarrollo de Ciencias Basicas (PEDECIBA) and Comision Sectorial de Investigacion Cientifica (Uruguay), and P.M. was supported by the Fonds National de la Recherche Scientifique and the Interuniversity Attraction Pole program of the Belgian government.
[1] Vertical-Cavity Surface-Emitting Lasers, edited by K.D. Choquette and D. G. Deppe, SPIE Proc. Vol. 3003 (SPIE, Bellingham, WA, 1997).

[2] M. San Miguel, in Semiconductor Quantum Optoelectronics, edited by A. Miller, M. Ebrahimzadeh, and D.M. Finlayson (Institute of Physics, Bristol, 1999), p. 339.

[3] A. Valle, J. Sarma, and K.A. Shore, Opt. Commun. 115, 297
(1995).

[4] A. Valle, J. Sarma, and K.A. Shore, IEEE J. Quantum Electron. 31, 1423 (1995).

[5] M. San Miguel, Q. Feng, and J.V. Moloney, Phys. Rev. A 52, 1728 (1995).

[6] J. Martin-Regalado, S. Balle, M. San Miguel, A. Valle, and L. Pesquera, Quantum Semiclassic. Opt. 9, 713 (1997). 
[7] J.Y. Law and G.P. Agrawal, Opt. Commun. 138, 95 (1997).

[8] T. Rossler, R.A. Indik, G.K. Harkness, J.V. Moloney, and C.Z. Ning, Phys. Rev. A 58, 3279 (1998).

[9] K. Panajotov, B. Ryvkin, J. Danckaert, M. Peeters, H. Thienpont, and I. Veretennicoff, IEEE Photonics Technol. Lett. 10, 6 (1998); B. Ryvkin, K. Panajotov, A. Georgievski, J. Danckaert, M. Peeters, G. Verschaffelt, H. Thienpont, and I. Veretennicoff, J. Opt. Soc. Am. B 16, 2106 (1999).

[10] S. Balle, E. Tolkachova, M. San Miguel, J.R. Tredicce, J. Martín-Regalado, and A. Gahl, Opt. Lett. 24, 1121 (1999).

[11] M.B. Willemsen, M.U.F. Khalid, M.P. Van Exter, and J.P. Woerdman, Phys. Rev. Lett. 82, 4815 (1999).

[12] S.P. Hegarty, G. Huyet, P. Porta, J.G. McInerney, K.D. Choquette, K.M. Geib, and H.Q. Hou, J. Opt. Soc. Am. B 16, 2060 (1999).

[13] T. Ackemann, S. Barland, M. Cara, S. Balle, J.R. Tredicce, R. Jager, M. Grabherr, M. Miller, and K.J. Ebeling, J. Opt. B: Quantum Semiclassical Opt. 2, 406 (2000).

[14] C. Degen, B. Krauskopf, G. Jennemann, I. Fischer, and W. Elsaßer, J. Opt. B: Quantum Semiclassical Opt. 2, 517 (2000).

[15] M.B. Willemsen, M.P. Van Exter, and J.P. Woerdman, Phys. Rev. Lett. 84, 4337 (2000).

[16] S. Barbay, G. Giacomelli, and F. Marin, Phys. Rev. E 61, 157 (2000); 63, 051110 (2001).

[17] L. Fratta, P. Debernardi, G.P. Bava, C. Degen, J. Kaiser, I. Fischer, and W. Elsaßer, Phys. Rev. A 64, 031803(R) (2001).

[18] J. Mulet, C.R. Mirasso, and M. San Miguel, Phys. Rev. A 64, 023817 (2001).

[19] J. Danckaert, B. Nagler, J. Albert, K. Panajotov, I. Veretennicoff, and T. Erneaux, Opt. Commun. 201, 129 (2002).

[20] J.S. Gustavsson, J.A. Vukuisic, J. Bengtsson, and Anders Larsson, IEEE J. Quantum Electron. 38, 203 (2002).

[21] Y.C. Chung and Y.H. Lee, IEEE Photonics Technol. Lett. 3, 597 (1991).

[22] J. Dellunde, A. Valle, and K.A. Shore, J. Opt. Soc. Am. B 13, 2477 (1996).
[23] J.Y. Law and G.P. Agrawal, IEEE J. Sel. Top. Quantum Electron. 3, 353 (1997).

[24] J.Y. Law and G.P. Agrawal, J. Opt. Soc. Am. B 15, 562 (1998).

[25] C. Masoller and N.B. Abraham, Phys. Rev. A 59, 3021 (1999).

[26] J. Dellunde, A. Valle, L. Pesquera, and K.A. Shore, J. Opt. Soc. Am. B 16, 2131 (1999).

[27] M. Giudici, S. Balle, T. Ackemann, S. Barland, and J.R. Tredicce, J. Opt. Soc. Am. B 16, 2114 (1999).

[28] D.W. Sukow, T. Heil, I. Fischer, A. Gavrielides, A. HohlAbiChedid, and W. Elsaßer, Phys. Rev. A 60, 667 (1999).

[29] R. Lang and K. Kobayashi, IEEE J. Quantum Electron. QE16, 347 (1980).

[30] F. Rogister, P. Mégret, O. Deparis, and M. Blondel, Phys. Rev. A 62, 061803(R) (2000).

[31] E.A. Viktorov and P. Mandel, Phys. Rev. Lett. 85, 3157 (2000).

[32] T.M. Carr, D. Pieroux, and P. Mandel, Phys. Rev. A 63, 033817 (2001).

[33] P. Mandel, Theoretical Problems in Cavity Nonlinear Optics (Cambridge University Press, Cambridge, England, 1997).

[34] B.A. Nguyen and P. Mandel, Opt. Commun. 112, 235 (1994).

[35] E.A. Viktorov and P. Mandel, Quantum Semiclassic. Opt. 8, 1205 (1996).

[36] M.S. Sodha and A.K. Ghatak, Inhomogeneous Optical Waveguides (Plenum Press, New York, 1977).

[37] M.S. Torre and H.F. Ranea-Sandoval, IEEE J. Quantum Electron. 36, 112 (2000).

[38] M.S. Torre and C. Masoller, Opt. Commun. 202, 311 (2002).

[39] K.Y. Lau, in Quantum Well Lasers, edited by P.S. Zory (Academic Press, Boston, 1993), pp. 217-275.

[40] W. Rideout, W.F. Sharfin, E.S. Koteles, M.O. Vassell, and B. Elman, IEEE Photonics Technol. Lett. 3, 784 (1991).

[41] R. Nagarajan, T. Fukushima, S.W. Corzine, and J.E. Bowers, Appl. Phys. Lett. 59, 1835 (1991).

[42] A. Valle, Appl. Phys. Lett. 73, 1607 (1998).

[43] P. Mandel, Eur. Phys. J. D 8, 431 (2000). 